\title{
The Exponential Stability Result of an Euler-Bernoulli Beam Equation with Interior Delays and Boundary Damping
}

\author{
Peng-cheng Han, Yan-fang Li, Gen-qi Xu, and Dan-hong Liu \\ Department of Mathematics, Tianjin University, Tianjin 300350, China \\ Correspondence should be addressed to Yan-fang Li; fangyan123li@tju.edu.cn
}

Received 20 January 2016; Accepted 9 March 2016

Academic Editor: Honglei Xu

Copyright ( $) 2016$ Peng-cheng Han et al. This is an open access article distributed under the Creative Commons Attribution License, which permits unrestricted use, distribution, and reproduction in any medium, provided the original work is properly cited.

We study the exponential stability of Euler-Bernoulli beam with interior time delays and boundary damping. At first, we prove the well-posedness of the system by the $C_{0}$ semigroup theory. Next we study the exponential stability of the system by constructing appropriate Lyapunov functionals. We transform the exponential stability issue into the solvability of inequality equations. By analyzing the relationship between delays parameters $\alpha$ and damping parameters $\beta$, we describe $(\beta, \alpha)$-region for which the system is exponentially stable. Furthermore, we obtain an estimation of the decay rate $\lambda^{*}$.

\section{Introduction}

It is well known that the time delay always exists in real system, which may be caused by acquisition of response and excitation data, online data processing, and computation of control forces. Since time delay may destroy stability $[1,2]$ even if it is very small, the stabilization problem of systems with time delays has been a hot topic in the mathematical control theory and engineering. In recent years, the systems described by PDEs with time delays have been an active area of research; see [3-7] and references therein. Generally speaking, there are mainly three kinds of time delay in the system, one is the interior time delay of the system (also called structural memory), one is the input delay (control delay), and the third is the output delay (measurement delay). Many scholars have made great efforts to minimize the negative effects of time delays although time delay cannot be eliminated due to its inherent nature, for example, [8-10] for boundary control with delays, $[11,12]$ for internal control delays, and [13] for output delays.

In past several years, the research on the Euler-Bernoulli beam with time delay has made great progress. For example, Park et al. [14] considered the stabilization problem of an Euler-Bernoulli beam with structural memory; Liang et al. [15] introduced the modified Smith predictor to EulerBernoulli beam with the boundary control and the delayed boundary measurement; Shang et al. [16-18] investigated the stabilization problem of the Euler-Bernoulli beam with boundary input delay; Yang et al. $[19,20]$ solved the stabilization problem of constant and variable coefficients EulerBernoulli beam with delayed observation and boundary control; at the same time, Jin and Guo [21] solved the output feedback stabilization of Euler-Bernoulli beam by Lyapunov approach. However, few people investigate the influence of an Euler-Bernoulli beam with interior delays and boundary damping on the system stability. In this paper we mainly study the exponential stability of a system described by the EulerBernoulli beam with interior delays and boundary damping. More precisely, we consider the following system, whose dynamic behavior is governed by the Euler-Bernoulli beam:

$$
\begin{aligned}
& y_{t t}(x, t)+y_{x x x x}(x, t)-2 \alpha y_{t}(x, t-\tau)=0, \\
& \quad x \in(0,1), t>0, \\
& y(0, t)=y_{x}(0, t)=y_{x x}(1, t)=0, \\
& y_{x x x}(1, t)=\beta y_{t}(1, t), \\
& y(x, 0)=y_{0}(x), \quad x \in(0,1), \\
& y_{t}(x, 0)=y_{1}(x), \\
& y_{t}(x, s)=h_{0}(x, s), \quad x \in(0,1), s \in(-\tau, 0),
\end{aligned}
$$


with $\beta, \alpha, \tau>0$, where $y_{t}(x, t)=\partial y / \partial t, y_{x}(x, t)=\partial y / \partial x$, and $\tau$ is the delay time. We mainly investigate its exponential stability.

The rest is organized as follows: In Section 2, we at first formulate problem (1) into an appropriate Hilbert space $\mathscr{H}$ and then study the well-posedness of the system by the semigroup theory. In Section 3, we construct a Lyapunov functional for system (1) and prove the exponential stability under certain conditions. By optimization parameters we obtain a complicated relationship between the decay rate $\lambda$ and delay time $\tau$. Finally, in Section 4, we give a brief conclusion.

\section{Well-Posedness of the System}

In this section, we will discuss the well-posedness and some basic properties of system (1). For the purpose, firstly we formulate system (1) into an appropriate Hilbert space.

Set

$$
\begin{aligned}
& z(x, \rho, t)=y_{t}(x, t-\tau \rho), \\
& \qquad x \in(0,1), \rho \in(0,1), t>0 .
\end{aligned}
$$

Clearly, $z(x, \rho, t)$ satisfies

$$
\begin{aligned}
\tau z_{t}(x, \rho, t)+z_{\rho}(x, \rho, t) & =0, \quad x \in(0,1), \rho \in(0,1), \\
z(x, 0, t) & =y_{t}(x, t) \\
z(x, 1, t) & =y_{t}(x, t-\tau) .
\end{aligned}
$$

Thus, system (1) is equivalent to the following:

$$
\begin{aligned}
& y_{t t}(x, t)+y_{x x x x}(x, t)-2 \alpha z(x, 1, t)=0, \quad x \in(0,1), \\
& \tau z_{t}(x, \rho, t)+z_{\rho}(x, \rho, t)=0, \quad x \in(0,1), \rho(0,1), \\
& y(0, t)=y_{x}(0, t)=y_{x x}(1, t)=0, \\
& y_{x x x}(1, t)=\beta y_{t}(1, t),
\end{aligned}
$$

$$
\begin{aligned}
& z(x, 0, t)=y_{t}(x, t), \\
& y(x, 0)=y_{0}(x), \\
& y_{t}(x, t)=y_{1}(x), \\
& z(x, \rho, 0)=z_{0}(x, \rho)=h_{0}(x,-\tau \rho) .
\end{aligned}
$$

Set

$$
H_{E}^{2}(0,1)=\left\{y \in H^{2}(0,1) \mid y(0)=y^{\prime}(0)=0\right\},
$$

where $H^{k}(0,1)$ is the usual Sobolev space of order $k$. We take the state space as

$$
\mathscr{H}=H_{E}^{2}(0,1) \times L^{2}(0,1) \times L^{2}[(0,1) \times(0,1)]
$$

equipped with the following inner product, for any $Y_{i}=$ $\left(f_{i}, g_{i}, h_{i}\right)^{T} \in \mathscr{H}, i=1,2$ :

$$
\begin{aligned}
\left\langle Y_{1}, Y_{2}\right\rangle_{\mathscr{H}}= & \int_{0}^{1}\left(f_{1, x x}(x) \overline{f_{2, x x}(x)}+g_{1}(x) \overline{g_{2}(x)}\right) d x \\
& +\tau \iint_{0}^{1} h_{1}(x, \rho) \overline{h_{2}(x, \rho)} d \rho d x .
\end{aligned}
$$

Obviously $\left(\mathscr{H},\|\cdot\|_{\mathscr{H}}\right)$ is a Hilbert space.

We define an operator $\mathscr{A}$ in $\mathscr{H}$ by

$$
\mathscr{A}\left(\begin{array}{l}
f \\
g \\
h
\end{array}\right)=\left(\begin{array}{c}
g(x) \\
-f_{x x x x}(x)+2 \alpha h(x, 1) \\
-\frac{1}{\tau} h_{\rho}(x, \rho)
\end{array}\right)
$$

with domain

$$
D(\mathscr{A})=\left\{\begin{array}{l|l}
(f, g, h)^{T} \in \mathscr{H} & \begin{array}{l}
f \in H^{4}[0,1] \cap H_{E}^{2}[0,1], g \in H_{E}^{2}(0,1), h \in H^{1}(0,1) \\
f^{\prime \prime}(1)=0, f^{\prime \prime \prime}(1)=\beta g(1), h(x, 0)=g(x)
\end{array}
\end{array}\right\} .
$$

With the assistance of operator $\mathscr{A}$, we can rewrite (4) as an evolution equation in $\mathscr{H}$ :

$$
\begin{aligned}
\frac{d Y(t)}{d t} & =\mathscr{A} Y(t), \quad t>0, \\
Y(0) & =Y_{0},
\end{aligned}
$$

where $Y(t)=\left(y(x, t), y_{t}(x, t), z(x, \rho, t)\right)^{T}$ and $Y_{0}=\left(y_{0}(x)\right.$, $\left.y_{1}(x), z_{0}(x, \rho)\right)^{T}$.

For operator $\mathscr{A}$, we have the following result.

Lemma 1. Let $\mathscr{A}$ be defined as (8) and (9). Then $\mathscr{A}$ is a closed and densely defined linear operator in $\mathscr{H}$. For any $\beta>0$ and $\alpha>0,0 \in \rho(A)$ and $\mathscr{A}^{-1}$ is compact on $\mathscr{H}$. Hence $\sigma(\mathscr{A})$ consists of all isolated eigenvalues of finite multiplicity.

Proof. It is easy to check that $\mathscr{A}$ is a closed and densely defined linear operator in $\mathscr{H}$; the detail of the verification is omitted.

Let $\alpha>0, \beta>0$ and for any $F \triangleq(\mu, \nu, \omega)^{T} \in \mathscr{H}$ we consider the equation $\mathscr{A} Y=F$ where $Y=(f, g, h) \in D(\mathscr{A})$; that is,

$$
\begin{aligned}
g(x) & =\mu(x), \\
-f_{x x x x}(x)+2 \alpha h(x, 1) & =v(x),
\end{aligned}
$$




$$
-\frac{1}{\tau} h_{\rho}(x, \rho)=\omega(x, \rho)
$$

with boundary condition

$$
f(0)=f^{\prime}(0)=f^{\prime \prime}(1)=0,
$$

$$
\begin{aligned}
g(x) & =\mu(x), \\
h(x, \rho) & =\mu(x)-\int_{0}^{\rho} \tau \omega(x, s) d s, \\
f(x) & =-\int_{0}^{x} \int_{0}^{r} \int_{1}^{q} \int_{1}^{p}\left(2 \alpha \mu(s)-2 \alpha \int_{0}^{1} \tau \omega(s, k) d k-v(s)\right) d s d p d q d r+\frac{1}{6} \beta \mu(1) x^{3} .
\end{aligned}
$$

Let $f, g, h$ be given as (13). Then we have $\mathscr{A} Y=F$ and $Y=(f, g, h) \in D(\mathscr{A})$. The closed operator theorem asserts that $0 \in \rho(A)$, and $\mathscr{A}^{-1}: \mathscr{H} \rightarrow D(\mathscr{A})$ is a bounded linear operator. Since $D(\mathscr{A}) \subset H_{E}^{4}(0,1) \times H^{2}(0,1) \times H^{1}(0,1)$, the Sobolev Embedding Theorem asserts that $\mathscr{A}^{-1}$ is a compact operator on $\mathscr{H}$. Hence, by the spectral theory of compact operator, $\sigma(\mathscr{A})$ consists of all isolated eigenvalues of finite multiplicity.

Theorem 2. Let $\mathscr{A}$ and $\mathscr{H}$ be defined as before. Then $\mathscr{A}$ generates a $C_{0}$ semigroup on $\mathscr{H}$. Hence, system (10) is well posed.

Proof. For any real $Y=(f, g, h)^{T} \in \mathscr{D}(\mathscr{A})$, we calculate

$$
\begin{aligned}
\langle\mathscr{A} Y, Y\rangle_{\mathscr{H}}= & \int_{0}^{1} g^{\prime \prime}(x) f^{\prime \prime}(x) d x \\
& +\int_{0}^{1} g(x)\left(-f_{x x x x}(x)+2 \alpha h(x, 1)\right) d x \\
& -\iint_{0}^{1} h_{\rho}(x, \rho) h(x, \rho) d x d \rho \\
= & -g(1) f^{\prime \prime \prime}(1)+2 \alpha \int_{0}^{1} h(x, 1) g(x) d x \\
& -\frac{1}{2} \int_{0}^{1}\left(h^{2}(x, 1)-h^{2}(x, 0)\right) d x \\
= & -\beta g^{2}(1)+2 \alpha \int_{0}^{1} h(x, 1) g(x) d x \\
& -\frac{1}{2} \int_{0}^{1} h^{2}(x, 1) d x+\frac{1}{2} \int_{0}^{1} g^{2}(x) d x .
\end{aligned}
$$

Since $\beta>0$, we have

$$
\begin{aligned}
\langle\mathscr{A} Y, Y\rangle_{\mathscr{H}} \leq & -\frac{1}{2} \int_{0}^{1}(h(x, 1)-2 \alpha g(x))^{2} d x \\
& +\frac{1}{2} \int_{0}^{1}\left(4 \alpha^{2}+1\right) g^{2}(x) d x \\
\leq & M\langle Y, Y\rangle_{\mathscr{H}},
\end{aligned}
$$

where $M=2 \alpha^{2}+1 / 2$, which shows that $\mathscr{A}-M I$ is a dissipative operator. This together with Lemma 1 shows that $\mathscr{A}-M I$ satisfies the conditions of Lumer-Phillips theorem [22]. So $\mathscr{A}$ generates a $C_{0}$ semigroup on $\mathscr{H}$.

\section{Exponential Stability of the System}

In this section, we consider the exponential stability issue of system (1) based on Lyapunov method.

The energy function of system (1) is defined as

$$
E(t)=\frac{1}{2} \int_{0}^{1}\left[y_{x x}^{2}(x, t)+y_{t}^{2}(x, t)\right] d x
$$

In what follows, we will give some lemmas that are the foundation of our method.

Lemma 3 (see [23]). Let $E(t)$ be a nonnegative function on $\mathbb{R}_{+}$. If there exists a function $V(t)$ and some positive numbers $c_{1}$ and $\lambda$ such that the conditions

$$
\begin{aligned}
& V(t)>c_{1} e^{\lambda t} E(t), \quad \forall t \geq 0, \\
& \dot{V}(t) \leq 0 \quad \forall t \geq 0
\end{aligned}
$$

hold, then $E(t)$ decays exponentially at rate $\lambda$.

In order to construct a function $V(t)$ satisfying the conditions in Lemma 3, we set

$$
G(t)=\eta \int_{0}^{1} x y_{x}(x, t) y_{t}(x, t) d x,
$$

where $\eta$ is a constant and satisfies $0<\eta<2$.

We can establish an equivalence relation between $G(t)$ and $E(t)$ via the following Lemma.

Lemma 4. Let $E(t)$ and $G(t)$ be defined as before. Then there exist positive constants $c_{2}$ and $c_{3}$ such that

$$
c_{2} E(t) \leq G(t)+E(t) \leq c_{3} E(t), \quad \forall t \geq 0,
$$

holds. 
Proof. Let $y(x, t)$ be the solution of (1). Applying Young's and Poincaré's inequalities

$$
\begin{aligned}
& \left|\int_{0}^{1} x y_{x}(x, t) y_{t}(x, t) d x\right| \\
& \quad \leq \frac{\delta}{2} \int_{0}^{1} y_{t}^{2}(x, t) d x+\frac{1}{2 \delta} \int_{0}^{1} x^{2} y_{x}^{2}(x, t) d x \\
& \quad \leq \frac{\delta}{2} \int_{0}^{1} x y_{t}^{2}(x, t) d x+\frac{1}{2 \delta} \int_{0}^{1} x^{2} d x \int_{0}^{x} y_{x x}^{2}(s, t) d s \\
& \quad<\frac{\delta}{2} \int_{0}^{1} y_{t}^{2}(x, t) d x+\frac{1}{8 \delta} \int_{0}^{1} y_{x x}^{2}(x, t) d x
\end{aligned}
$$$$
\forall \delta>0, t \geq 0
$$

Taking $\delta=1 / 2$, we get

$$
\left|\eta \int_{0}^{1} x y_{x}(x, t) y_{t}(x, t) d x\right|<\frac{\eta}{2} E(t), \quad t \geq 0 .
$$

Since $0<\eta<2$, we can set $c_{1}=1-\eta / 2$ and $c_{2}=1+\eta / 2$; then

$$
c_{2} E(t) \leq G(t)+E(t) \leq c_{3} E(t) .
$$

The desired inequality follows.

Let $\lambda>0$. We define a function $V(t)$ by

$$
V(t)=V_{1}(t)+V_{2}(t)
$$

where

$$
\begin{aligned}
& V_{1}(t)=e^{2 \lambda t}\left(\frac{1}{2} \int_{0}^{1}\left(y_{x x}^{2}(x, t)+y_{t}^{2}(x, t)\right) d x\right. \\
& \left.+\eta \int_{0}^{1} x y_{x}(x, t) y_{t}(x, t) d x\right), \\
& V_{2}(t)=2 \alpha e^{-\lambda \tau} \int_{0}^{1} \int_{t-\tau}^{t} e^{2 \lambda(s+\tau)} y_{t}^{2}(x, s) d s d x .
\end{aligned}
$$

Noting that $\alpha>0$, according to Lemma 4 we can see that the following result is true.

Lemma 5. Let $V(t)$ defined as before. Then $V(t)$ satisfies condition (17); that is,

$$
V(t)>c_{2} e^{2 \lambda t} E(t), \quad t \geq 0 .
$$

In what follows, we calculate $\dot{V}(t)$. For $V_{1}(t)$ we have the following result.

Lemma 6. Let $V_{1}(t)$ be defined as before and let $y(x, t)$ be the solution of (1). Then

$$
\begin{gathered}
\dot{V}_{1}(t) \leq e^{2 \lambda t}\left[\left(\lambda-\frac{3 \eta}{2}+\frac{\lambda \eta}{2}+\frac{\beta \eta}{2}+\frac{\alpha \eta^{2}}{4} e^{\lambda \tau}\right)\right. \\
\cdot \int_{0}^{1} y_{x x}^{2} d x+\left(\lambda-\frac{\eta}{2}+\frac{\lambda \eta}{2}+\alpha e^{\lambda \tau}\right)
\end{gathered}
$$

$$
\begin{aligned}
& \left.\cdot \int_{0}^{1} y_{t}^{2}(x, t) d x\right]+e^{2 \lambda t}\left(-\beta+\frac{\eta}{2}+\frac{\beta \eta}{2}\right) y_{t}^{2}(1, t) \\
& +2 \alpha e^{-\lambda \tau} e^{\lambda t} \int_{0}^{1} y_{t}^{2}(x, t-\tau) d x
\end{aligned}
$$

Proof. By definition, we see that

$$
V_{1}(t)=e^{2 \lambda t}(E(t)+G(t)),
$$

where $E(t)$ and $G(t)$ are defined as before.

So

$$
\dot{V}_{1}(t)=e^{2 \lambda t}(2 \lambda(E(t)+G(t))+\dot{E}(t)+\dot{G}(t)) .
$$

In what follows, we will calculate $\dot{E}(t)$ and $\dot{G}(t)$.

Using integration by parts and the boundary condition, we have

$$
\begin{aligned}
& \dot{E}(t)=\int_{0}^{1} y_{x x}(x, t) y_{x x t}(x, t)+y_{t}(x, t) y_{t t}(x, t) d x \\
& =\left.y_{x x}(x, t) y_{t x}(x, t)\right|_{0} ^{1} \\
& -\int_{0}^{1} y_{x x x}(x, t) y_{t x}(x, t) d x \\
& -\int_{0}^{1} y_{t}(x, t) y_{x x x x}(x, t) d x \\
& +\int_{0}^{1} 2 \alpha y_{t}(x, t) y_{t}(x, t-\tau) d x \\
& =\left.y_{x x}(x, t) y_{t x}(x, t)\right|_{0} ^{1}-\left.y_{x x x}(x, t) y_{t}(x, t)\right|_{0} ^{1} \\
& +\int_{0}^{1} y_{x x x x}(x, t) y_{t}(x, t) d x \\
& -\int_{0}^{1} y_{t}(x, t) y_{x x x x}(x, t) d x \\
& +2 \alpha \int_{0}^{1} y_{t}(x, t) y_{t}(x, t-\tau) d x=-\beta y_{t}^{2}(1, t) \\
& +2 \alpha \int_{0}^{1} y_{t}(x, t) y_{t}(x, t-\tau) d x, \\
& \dot{G}(t)=\eta\left(\int_{0}^{1} x y_{t x}(x, t) y_{t}(x, t) d x\right. \\
& \left.+\int_{0}^{1} x y_{x}(x, t) y_{t t}(x, t) d x\right) \\
& =\eta\left(\int_{0}^{1} x y_{t x}(x, t) y_{t}(x, t) d x\right. \\
& +2 \alpha \int_{0}^{1} x y_{x}(x, t) y_{t}(x, t-\tau) d x
\end{aligned}
$$


Journal of Difference Equations

5

$$
\begin{aligned}
& \left.-\int_{0}^{1} x y_{x}(x, t) y_{x x x}(x, t) d x\right)=\eta\left(\frac{1}{2} y_{t}^{2}(1, t)\right. \\
& -\frac{1}{2} \int_{0}^{1} y_{t}^{2}(x, t) d x-\frac{3}{2} \int_{0}^{1} y_{x x}^{2}(x, t) d x \\
& -\beta y_{x}(1, t) y_{t}(1, t) \\
& \left.+2 \alpha \int_{0}^{1} x y_{x}(x, t) y_{t}(x, t-\tau) d x\right),
\end{aligned}
$$

where we have used equalities

$$
\begin{aligned}
& \int_{0}^{1} x y_{t x}(x, t) y_{t}(x, t) d x \\
& \quad=\frac{1}{2} y_{t}^{2}(1, t)-\frac{1}{2} \int_{0}^{1} y_{t}^{2}(x, t) d x \\
& \int_{0}^{1} x y_{x x x x}(x, t) y_{x}(x, t) d x \\
& =\frac{3}{2} \int_{0}^{1} y_{x x}^{2}(x, t) d x+\beta y_{x}(1, t) y_{t}(1, t)
\end{aligned}
$$

Summarizing the above all, we have

$$
\begin{aligned}
& \dot{V}_{1}=e^{2 \lambda t}\left[\lambda \int_{0}^{1}\left(y_{x x}^{2}(x, t)+y_{t}^{2}(x, t)\right) d x\right. \\
& \left.+2 \lambda \eta \int_{0}^{1} x y_{x}(x, t) y_{t}(x, t) d x\right]+e^{2 \lambda t}\left[-\beta y_{t}^{2}(1, t)\right. \\
& +\frac{\eta}{2} y_{t}^{2}(1, t)-\beta \eta y_{x}(1, t) y_{t}(1, t) \\
& -\frac{\eta}{2} \int_{0}^{1} y_{t}^{2}(x, t) d x-\frac{3 \eta}{2} \int_{0}^{1} y_{x x}^{2}(x, t) d x \\
& +2 \alpha \int_{0}^{1} y_{t}(x, t) y_{t}(x, t-\tau) d x \\
& \left.+2 \alpha \eta \int_{0}^{1} x y_{x}(x, t) y_{t}(x, t-\tau) d x\right] \\
& =e^{2 \lambda t}\left[\left(\lambda-\frac{3 \eta}{2}\right) \int_{0}^{1} y_{x x}^{2}(x, t) d x\right. \\
& \left.+\left(\lambda-\frac{\eta}{2}\right) \int_{0}^{1} y_{t}^{2}(x, t) d x\right] \\
& +e^{2 \lambda t}\left[2 \lambda \eta \int_{0}^{1} x y_{x}(x, t) y_{t}(x, t) d x\right. \\
& +2 \alpha \int_{0}^{1} y_{t}(x, t) y_{t}(x, t-\tau) d x \\
& \left.+2 \alpha \eta \int_{0}^{1} x y_{x}(x, t) y_{t}(x, t-\tau) d x\right] \\
& +e^{2 \lambda t}\left[\frac{\eta-2 \beta}{2} y_{t}^{2}(1, t)-\beta \eta y_{x}(1, t) y_{t}(1, t)\right] \text {. }
\end{aligned}
$$

Since

$$
\begin{aligned}
& \int_{0}^{1} x y_{x}(x, t) y_{t}(x, t) d x \\
& \quad \leq \frac{1}{4} \int_{0}^{1} y_{x x}^{2} d x+\frac{1}{4} \int_{0}^{1} y_{t}^{2}(x, t) d x, \\
& -\beta \eta y_{x}(1, t) y_{t}(1, t) \leq \frac{\beta \eta}{2} y_{x}^{2}(1, t)+\frac{\beta \eta}{2} y_{t}^{2}(1, t) \\
& \quad \leq \frac{\beta \eta}{2} \int_{0}^{1} y_{x x}^{2}(x, t) d x+\frac{\beta \eta}{2} y_{t}^{2}(1, t),
\end{aligned}
$$

we have

$$
\begin{aligned}
\dot{V}_{1} & \leq e^{2 \lambda t}\left[\left(\lambda-\frac{3 \eta}{2}+\frac{\lambda \eta}{2}\right) \int_{0}^{1} y_{x x}^{2}(x, t) d x\right. \\
& \left.+\left(\lambda-\frac{\eta}{2}+\frac{\lambda \eta}{2}\right) \int_{0}^{1} y_{t}^{2}(x, t) d x\right] \\
& +e^{2 \lambda t}\left[2 \alpha \int_{0}^{1} y_{t}(x, t) y_{t}(x, t-\tau) d x\right. \\
& \left.+2 \alpha \eta \int_{0}^{1} x y_{x}(x, t) y_{t}(x, t-\tau) d x\right]+e^{2 \lambda t} \frac{\eta-2 \beta}{2} \\
& +y_{t}^{2}(1, t)+e^{2 \lambda t}\left[\frac{\beta \eta}{2} \int_{0}^{1} y_{x x}^{2}(x, t) d x\right. \\
& \left.+\frac{\beta \eta}{2} y_{t}^{2}(1, t)\right] \\
& =e^{2 \lambda t}\left[\left(\lambda-\frac{3 \eta}{2}+\frac{\lambda \eta}{2}+\frac{\beta \eta}{2}\right) \int_{0}^{1} y_{x x}^{2}(x, t) d x\right. \\
& \left.+\left(\lambda-\frac{\eta}{2}+\frac{\lambda \eta}{2}\right) \int_{0}^{1} y_{t}^{2}(x, t) d x\right] \\
& \left.+2 \alpha \eta \int_{0}^{1} x y_{x}(x, t) y_{t}(x, t-\tau) d x\right]+e^{2 \lambda t} \\
& +2 \beta+\beta \eta \\
& +2 \alpha \int_{0}^{1} y_{t}^{2}(x, t) y_{t}(x, t-\tau) d x
\end{aligned}
$$

We now estimate the integral terms with time delay. Applying Young's and Poincaré's inequalities, we have

$$
\begin{aligned}
& \int_{0}^{1} y_{t}(x, t) y_{t}(x, t-\tau) d x \\
& \quad \leq \frac{\delta_{1}}{2} \int_{0}^{1} y_{t}^{2}(x, t-\tau) d x+\frac{1}{2 \delta_{1}} \int_{0}^{1} y_{t}^{2}(x, t) d x \\
& \int_{0}^{1} x y_{x}(x, t) y_{t}(x, t-\tau) d x \\
& \quad \leq \frac{\delta_{2}}{2} \int_{0}^{1} y_{t}^{2}(x, t-\tau) d x+\frac{1}{8 \delta_{2}} \int_{0}^{1} y_{x x}^{2}(x, t) d x .
\end{aligned}
$$


Thus,

$$
\begin{aligned}
& \dot{V}_{1}(t) \leq e^{2 \lambda t}\left[\left(\lambda-\frac{3 \eta}{2}+\frac{\lambda \eta}{2}+\frac{\beta \eta}{2}+\frac{\alpha \eta}{4 \delta_{2}}\right) \int_{0}^{1} y_{x x}^{2} d x\right. \\
& \left.+\left(\lambda-\frac{\eta}{2}+\frac{\lambda \eta}{2}+\frac{\alpha}{\delta_{1}}\right) \int_{0}^{1} y_{t}^{2}(x, t) d x\right]+e^{2 \lambda t}(-\beta \\
& \left.+\frac{\eta}{2}+\frac{\beta \eta}{2}\right) y_{t}^{2}(1, t)+\left[\alpha \delta_{1}+\alpha \eta \delta_{2}\right] \\
& \cdot e^{2 \lambda t} \int_{0}^{1} y_{t}^{2}(x, t-\tau) d x .
\end{aligned}
$$

Taking $\delta_{1}=e^{-\lambda \tau}, \delta_{2}=e^{-\lambda \tau} / \eta$, we obtain

$$
\begin{aligned}
& \dot{V}_{1}(t) \\
& \leq e^{2 \lambda t}\left[\left(\lambda-\frac{3 \eta}{2}+\frac{\lambda \eta}{2}+\frac{\beta \eta}{2}+\frac{\alpha \eta^{2}}{4} e^{\lambda \tau}\right) \int_{0}^{1} y_{x x}^{2} d x\right. \\
& \left.+\left(\lambda-\frac{\eta}{2}+\frac{\lambda \eta}{2}+\alpha e^{\lambda \tau}\right) \int_{0}^{1} y_{t}^{2}(x, t) d x\right] \\
& +e^{2 \lambda t}\left(-\beta+\frac{\eta}{2}+\frac{\beta \eta}{2}\right) y_{t}^{2}(1, t) \\
& +2 \alpha e^{-\lambda \tau} e^{2 \lambda t} \int_{0}^{1} y_{t}^{2}(x, t-\tau) d x
\end{aligned}
$$

The desired inequality follows.

Since

$$
V_{2}(t)=2 \alpha e^{-\lambda \tau} \int_{0}^{1} \int_{t-\tau}^{t} e^{2 \lambda(s+\tau)} y_{t}^{2}(x, s) d s d x
$$

we have

$$
\begin{aligned}
\dot{V}_{2}(t)= & 2 \alpha e^{-\lambda \tau} \int_{0}^{1} e^{2 \lambda(t+\tau)} y_{t}^{2}(x, t) d x \\
& -2 \alpha e^{-\lambda \tau} \int_{0}^{1} e^{2 \lambda t} y_{t}^{2}(x, t-\tau) d x .
\end{aligned}
$$

Employing the estimate, we have

$$
\begin{aligned}
& \dot{V}(t)=\dot{V}_{1}(t)+\dot{V}_{2}(t) \\
& \leq e^{2 \lambda t}\left[\left(\lambda-\frac{3 \eta}{2}+\frac{\lambda \eta}{2}+\frac{\beta \eta}{2}+\frac{\alpha \eta^{2}}{4} e^{\lambda \tau}\right) \int_{0}^{1} y_{x x}^{2} d x\right. \\
&\left.+\left(\lambda-\frac{\eta}{2}+\frac{\lambda \eta}{2}+3 \alpha e^{\lambda \tau}\right) \int_{0}^{1} y_{t}^{2}(x, t) d x\right] \\
&+e^{2 \lambda t}\left(-\beta+\frac{\eta}{2}+\frac{\beta \eta}{2}\right) y_{t}^{2}(1, t) .
\end{aligned}
$$

Clearly, if the parameters $\eta, \beta, \alpha, \lambda$, and $\tau$ are such that the inequalities

$$
\begin{aligned}
\lambda-\frac{3 \eta}{2}+\frac{\lambda \eta}{2}+\frac{\beta \eta}{2}+\frac{\alpha \eta^{2} e^{\lambda \tau}}{4} & \leq 0 \\
\lambda-\frac{\eta}{2}+\frac{\lambda \eta}{2}+3 \alpha e^{\lambda \tau} & \leq 0 \\
-\beta+\frac{\eta}{2}+\frac{\beta \eta}{2} & \leq 0
\end{aligned}
$$

hold, then we have $\dot{V}(t) \leq 0$.

Summarizing discussion above, we have the following result.

Theorem 7. Let $y(x, t)$ be the solution of (1), and let $0<\eta<2$ and $\lambda>0$. If inequalities (41) hold, then the energy function $E(t)$ decays exponentially at rate $2 \lambda$.

We now are in a proposition to study the solvability of inequalities (41). Noting that $\eta$ is not a system parameter, it is only a middle parameter which is introduced in the multiplier term. From the third inequality of (41) we see that $\eta$ and $\beta$ have a relationship:

$$
0<\eta<\frac{2 \beta}{1+\beta} .
$$

Taking $\eta=2 \beta /(1+\beta)$, (41) is equivalent to

$$
\begin{array}{r}
\lambda-\frac{3 \beta}{1+\beta}+\frac{\lambda \beta}{1+\beta}+\frac{\beta^{2}}{1+\beta}+\frac{\alpha \beta^{2} e^{\lambda \tau}}{(1+\beta)^{2}} \leq 0, \\
\lambda-\frac{\beta}{1+\beta}+\frac{\lambda \beta}{1+\beta}+3 \alpha e^{\lambda \tau} \leq 0 .
\end{array}
$$

Theorem 8. Set $\eta=2 \beta /(1+\beta)$. If $\alpha$ and $\beta$ satisfy the inequality

$$
\alpha<\min \left\{\frac{3}{\beta}+2-\beta, \frac{\beta}{3(1+\beta)}\right\},
$$

then there exists $\lambda^{*}>0$ such that for $\lambda \in\left(0, \lambda^{*}\right]$ inequality (41) holds true.

Proof. If (44) holds, then

$$
\begin{aligned}
& \alpha<\frac{3}{\beta}+2-\beta, \\
& \alpha<\frac{\beta}{3(1+\beta)},
\end{aligned}
$$

or equivalently

$$
\begin{array}{r}
-\frac{3 \beta}{1+\beta}+\frac{\beta^{2}}{1+\beta}+\frac{\alpha \beta^{2}}{(1+\beta)^{2}}<0, \\
-\frac{\beta}{1+\beta}+\frac{\lambda \beta}{1+\beta}+3 \alpha<0 .
\end{array}
$$


Set

$$
\begin{aligned}
& f(x)=x-\frac{3 \beta}{1+\beta}+\frac{x \beta}{1+\beta}+\frac{\beta^{2}}{1+\beta}+\frac{\alpha \beta^{2} e^{x \tau}}{(1+\beta)^{2}}, \\
& \quad x \geq 0, \\
& g(y)=y-\frac{\beta}{1+\beta}+\frac{y \beta}{1+\beta}+3 \alpha e^{y \tau}, \quad y \geq 0 .
\end{aligned}
$$

Since

$$
\begin{aligned}
& f(0)=-\frac{3 \beta}{1+\beta}+\frac{\beta^{2}}{1+\beta}+\frac{\alpha \beta^{2}}{(1+\beta)^{2}}<0, \\
& g(0)=-\frac{\beta}{1+\beta}+\frac{\lambda \beta}{1+\beta}+3 \alpha<0, \\
& f(3)=3+\frac{\beta^{2}}{1+\beta}+\frac{\alpha \beta^{2} e^{3 \tau}}{(1+\beta)^{2}}>0, \\
& g(1)=1+3 \alpha e^{\tau}>0,
\end{aligned}
$$

there exist $x^{*} \in(0,3)$ and $y^{*} \in(0,1)$ such that $f\left(x^{*}\right)=0$ and $g\left(y^{*}\right)=0$.

Set

$$
\begin{aligned}
& x_{*}=\min \left\{x^{*} \in(0,3), f\left(x^{*}\right)=0\right\}, \\
& y_{*}=\min \left\{y^{*} \in(0,1), g\left(y^{*}\right)=0\right\},
\end{aligned}
$$

and $\lambda^{*}=\min \left\{x_{*}, y_{*}\right\}$. Clearly, when $\lambda \in\left(0, \lambda^{*}\right]$, we have $f(\lambda) \leq 0$ and $g(\lambda) \leq 0$, so (43) holds. Hence (41) holds true.

In what follows, we discuss the property of the function

$$
G(\beta)=\min \left\{\frac{3}{\beta}+2-\beta, \frac{\beta}{3(1+\beta)}\right\}, \quad \beta \geq 0 .
$$

We consider equation

$$
\frac{3}{\beta}+2-\beta=\frac{\beta}{3(1+\beta)},
$$

and it is equivalent to

$$
9+15 \beta+2 \beta^{2}-3 \beta^{3}=0 .
$$

This equation has three real roots $\beta_{1}<\beta_{2}<0<2<\beta_{3}<3$. So we have

$$
\begin{aligned}
G(\beta) & = \begin{cases}\frac{\beta}{3(1+\beta)}, & \beta \in\left(0, \beta_{3}\right] \\
\frac{3}{\beta}+2-\beta, & \beta \in\left[\beta_{3}, 3\right] \\
\frac{3}{\beta}+2-\beta<0, & \beta \geq 3,\end{cases} \\
\max _{\beta>0} G(\beta) & =\frac{\beta_{3}}{3\left(1+\beta_{3}\right)} .
\end{aligned}
$$

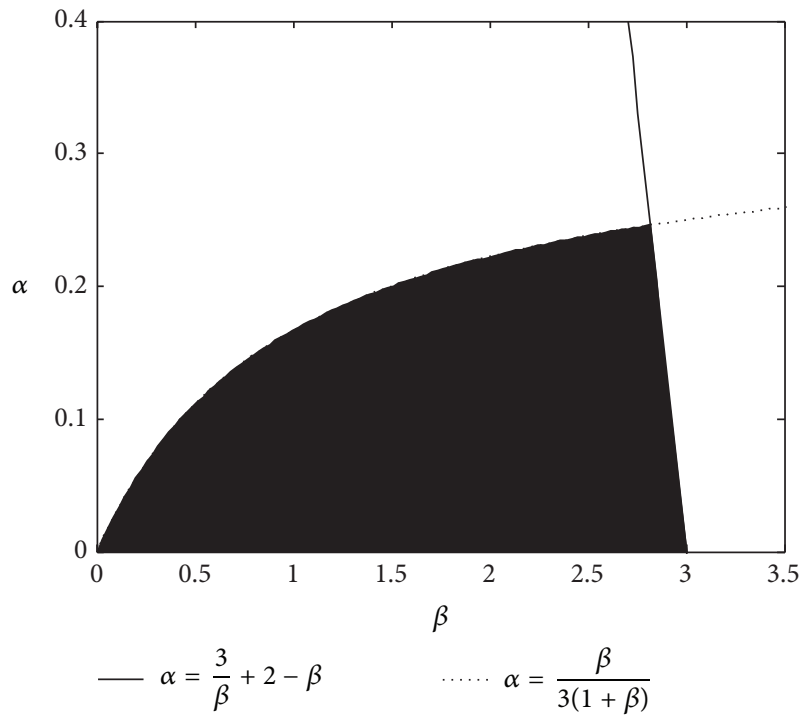

FIGURE 1: The graph of function $G$, which gives the relationship between $\alpha$ and $\beta$.

3.1. $(\beta, \alpha)$-Region of the Exponential Stability. According to (52) we determine $\beta_{3} \simeq 2.818$. And according to (53) we draw the $(\beta, \alpha)$-region.

The picture of $G(\beta)$ is given as Figure 1. $(\beta, \alpha)$-region is given by

$$
\sum(\beta, \alpha)=\{(\beta, \alpha) \mid \alpha<G(\beta), \beta \in(0,3)\} .
$$

Figure 1 gives the graph of function $G(\beta)$ that gives the relationship between $\alpha$ and $\beta$ with which system (1) is exponentially stable. From this picture we see that if $\alpha$ is larger, we cannot stabilize it by the boundary damping. $\alpha$ has upper bound $\alpha^{*}=\beta_{3} / 3\left(1+\beta_{3}\right) \simeq 0.246$.

3.2. The Best Decay Rate $\lambda^{*}$. Suppose that $\alpha<G(\beta)$ with $\beta \epsilon$ $(0,3)$. According to (43) we determine the best decay rate $\lambda^{*}$. Note that $\lambda^{*}=\min \left\{x_{*}, y_{*}\right\} \in(0,1)$, where $x_{*}$ and $y_{*}$ solve the following equation, respectively:

$$
\begin{array}{r}
x_{*}-\frac{3 \beta}{1+\beta}+\frac{x_{*} \beta}{1+\beta}+\frac{\beta^{2}}{1+\beta}+\frac{\alpha \beta^{2} e^{x_{*} \tau}}{(1+\beta)^{2}}=0, \\
y_{*}-\frac{\beta}{1+\beta}+\frac{y_{*} \beta}{1+\beta}+3 \alpha e^{y_{*} \tau}=0 .
\end{array}
$$

Firstly, let

$$
\begin{aligned}
& p(x)=x-\frac{3 \beta}{1+\beta}+\frac{x \beta}{1+\beta}+\frac{\beta^{2}}{1+\beta}+\frac{\alpha \beta^{2} e^{x \tau}}{(1+\beta)^{2}}, \\
& q(x)=x-\frac{\beta}{1+\beta}+\frac{x \beta}{1+\beta}+3 \alpha e^{x \tau} .
\end{aligned}
$$



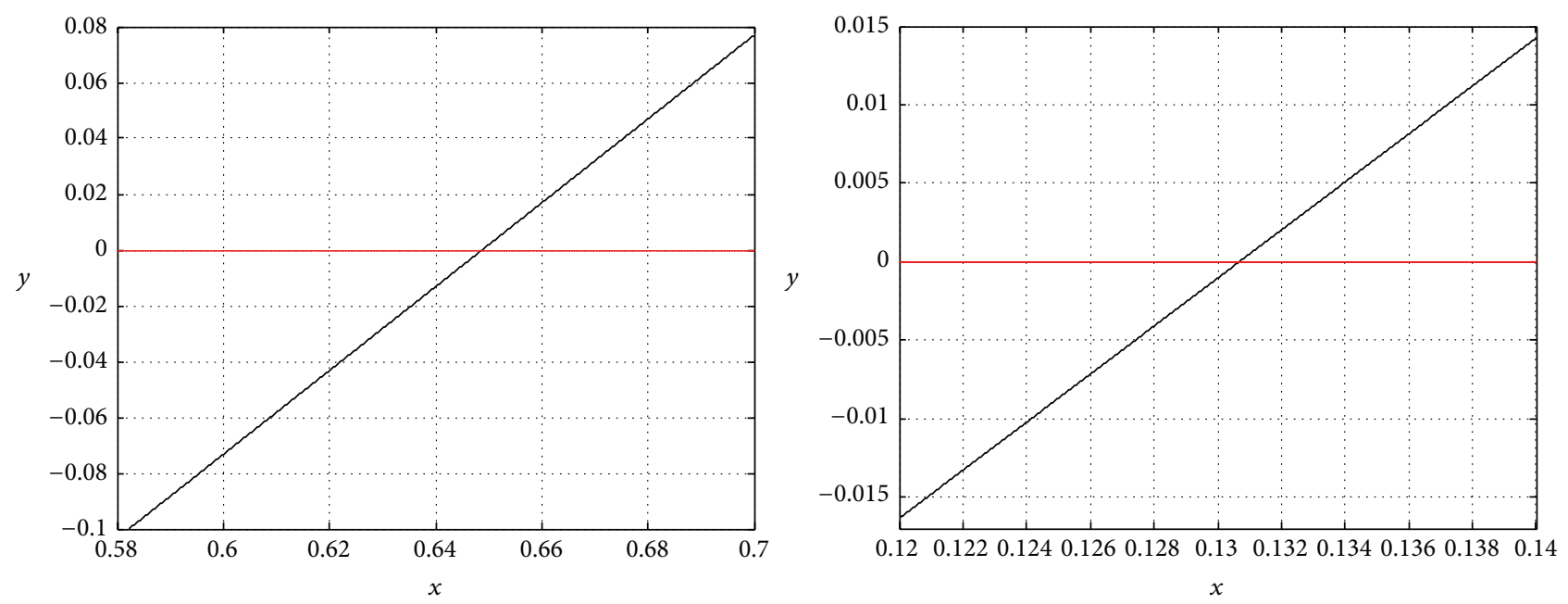

Figure $2: \beta=1, \alpha=0.1$, and $\tau=0.1$.

Obviously, $p(x)$ and $q(x)$ both are monotonic function. For comparing to the two equations of (56), we define the function $f(x)$ :

$$
\begin{aligned}
f(x)= & p(x)-q(x) \\
= & x-\frac{3 \beta}{1+\beta}+\frac{x \beta}{1+\beta}+\frac{\beta^{2}}{1+\beta}+\frac{\alpha \beta^{2} e^{x \tau}}{(1+\beta)^{2}} \\
& -\left(x-\frac{\beta}{1+\beta}+\frac{x \beta}{1+\beta}+3 \alpha e^{x \tau}\right) \\
= & \frac{\beta^{2}-2 \beta}{1+\beta}+\alpha\left[\frac{\beta^{2}}{(1+\beta)^{2}}-3\right] e^{x \tau} .
\end{aligned}
$$

Since $\beta, \alpha, \tau>0$, we have $\alpha\left[\beta^{2} /(1+\beta)^{2}-3\right] e^{x \tau}<0$. When $\beta \in(0,2]$, we get $\left(\beta^{2}-2 \beta\right) /(1+\beta)<0$; that is, $f(x)<0$. Therefore, when $\beta \in(0,2], \lambda^{*}=y_{*}$.

An example is given: Assume that $\beta=1, \alpha=0.1, \tau=0.1$ (i.e., they are constants). We obtain the approximative zero points of $p(x)$ and $q(x)$ from Figure 2. That is, $x_{*} \simeq 0.6489$ and $y_{*} \simeq 0.1307$. Therefore, we get the best decay rate $\lambda^{*}=$ $y_{*} \simeq 0.1307$.

Next, we consider $\beta \in\left(2, \beta_{3}\right)$. By researching (58), we can obtain $\left(\beta^{2}-2 \beta\right) /(1+\beta)>0$ and $\left[\beta^{2} /(1+\beta)^{2}-3\right] e^{x \tau} \leq$ $\beta^{2} /(1+\beta)^{2}-3 \leq 0$. We have $f(x) \leq\left(\beta^{2}-2 \beta\right) /(1+\beta)+$ $\alpha\left[\beta^{2} /(1+\beta)^{2}-3\right]$.

Let

$$
H=\frac{\beta^{2}-2 \beta}{1+\beta}+\alpha\left[\frac{\beta^{2}}{(1+\beta)^{2}}-3\right]<0 .
$$

We have $\alpha>\left(2 \beta-\beta^{2}\right)(1+\beta) /\left(\beta^{2}-3(1+\beta)^{2}\right)>0$. According to $(\beta, \alpha)$-region of the exponential stability, we obtain $\alpha<$ $\beta / 3(1+\beta)$. After comparison,

$$
\frac{\left(2 \beta-\beta^{2}\right)(1+\beta)}{\beta^{2}-3(1+\beta)^{2}}<\alpha<\frac{\beta}{3(1+\beta)} .
$$

To summarize, $\beta \in\left(2, \beta_{3}\right]$, when $\left(2 \beta-\beta^{2}\right)(1+\beta) /\left(\beta^{2}-\right.$ $\left.3(1+\beta)^{2}\right)<\alpha<\beta / 3(1+\beta)$, we can get $f(x) \leq H<0$. Therefore, the best decay $\lambda^{*}=y_{*}$.

Then, we give two examples.

Assume that $\beta=2.1 \in\left(2, \beta_{3}\right], \alpha=0.1 \in(0.0267,0.2258)$, $\tau=0.1$ (i.e., they are constants); we can obtain Figure 3. We get the best decay rate $\lambda^{*}=y_{*}$.

Assume that $\beta=2.1 \in\left(2, \beta_{3}\right], \alpha=0.2 \in(0.0267,0.2258)$, $\tau=0.1$ (i.e., they are constants); we can obtain Figure 4. We get the best decay rate $\lambda^{*}=y_{*}$.

Finally, we consider $\beta \in\left(\beta_{3}, 3\right)$. We can also obtain $\alpha>$ $\left(2 \beta-\beta^{2}\right)(1+\beta) /\left(\beta^{2}-3(1+\beta)^{2}\right)>0$, when $f(x) \leq H<0$. Here, $\alpha<3 / \beta+2-\beta$. After comparison,

$$
\frac{\left(2 \beta-\beta^{2}\right)(1+\beta)}{\beta^{2}-3(1+\beta)^{2}}>\frac{3}{\beta}+2-\beta .
$$

Therefore, Not all $f(x)<0$ were always correct.

Summarizing the above all, the best decay $\lambda^{*}$ is easy to determine, when $\beta \in(0,2)$. The best decay $\lambda^{*}$ is not easy to determine, when $\beta \in(2,3)$. We have two conclusion:

(i) $\beta \in(0,2]$, the best decay $\lambda^{*}=y_{*}$;

(ii) $\beta \in\left(2, \beta_{3}\right]$, when $\left(2 \beta-\beta^{2}\right)(1+\beta) /\left(\beta^{2}-3(1+\beta)^{2}\right)<$ $\alpha<\beta / 3(1+\beta)$, the best decay $\lambda^{*}=y_{*}$.

\section{Conclusions}

In this paper, using the Lyapunov functional approach we discussed the exponential stabilization of an Euler-Bernoulli beam equation with interior delays and boundary damping. Different from the earlier papers, we added a multiplier term $e^{2 \lambda t}$ to the Lyapunov function so as to transform the exponential stability. By solving the inequality equations, we give the exponential stability region of the system.

We note that the method used in this paper also can apply to the investigation of the exponential stability of other 

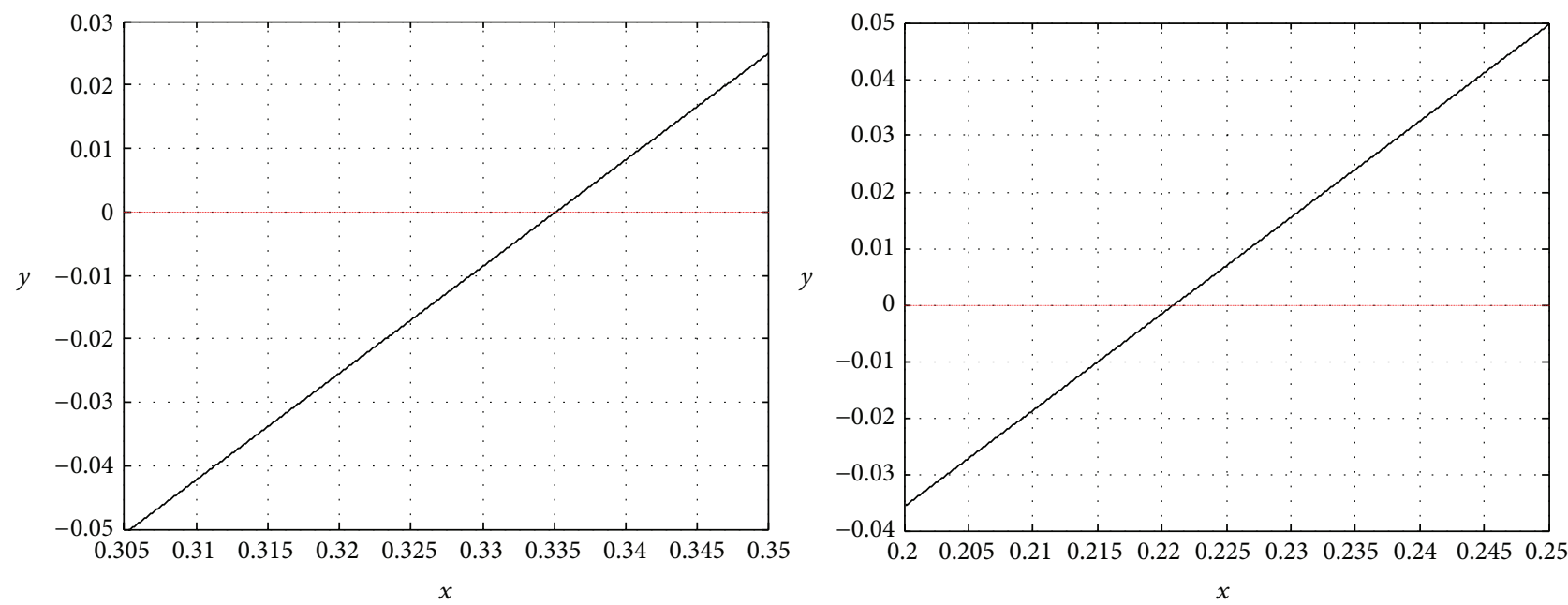

Figure 3: $\beta=2.1, \alpha=0.1$, and $\tau=0.1$.
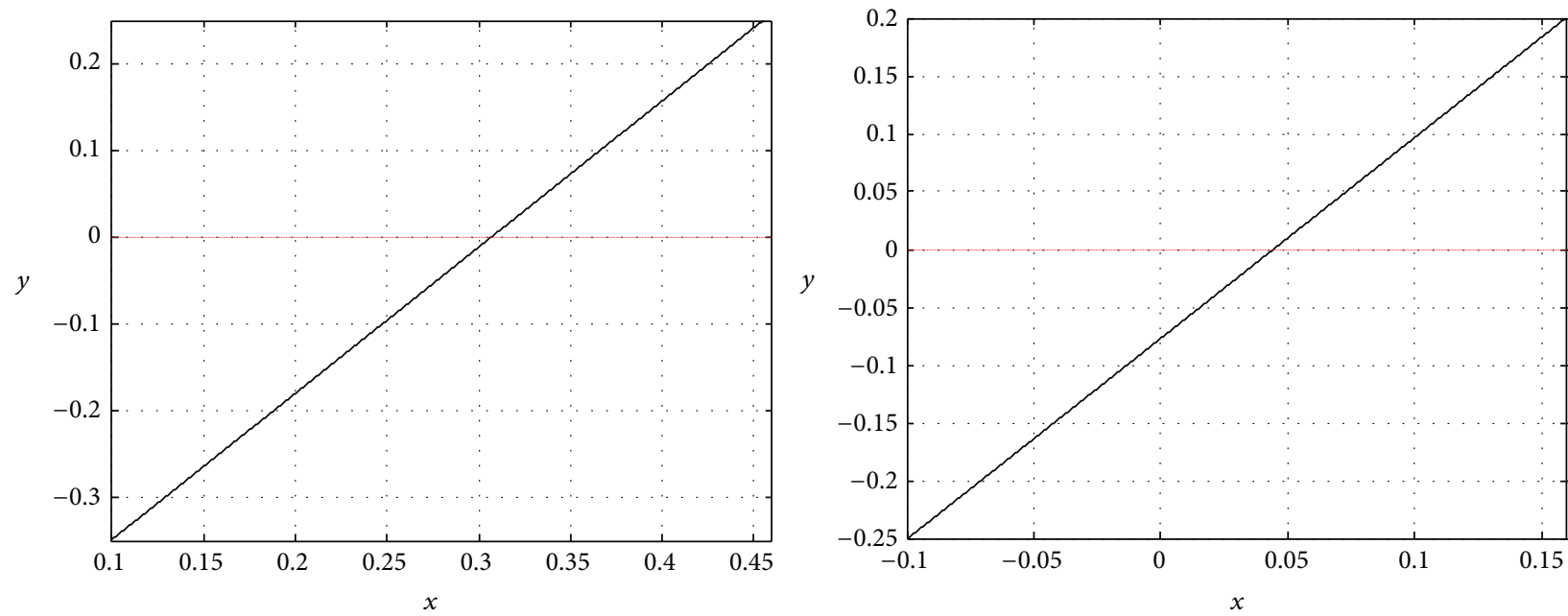

Figure 4: $\beta=2.1, \alpha=0.2$, and $\tau=0.1$.

model. In the future, we will study the boundary feedback control anti-interior time delay for other models.

\section{Competing Interests}

The authors declare that there are no competing interests regarding the publication of this paper.

\section{Acknowledgments}

This research is supported by the Natural Science Foundation of China (Grant nos. NSFC-61174080, 61573252, and 61503275).

\section{References}

[1] R. Datko, "Two questions concerning the boundary control of certain elastic systems," Journal of Differential Equations, vol. 92, no. 1, pp. 27-44, 1991.
[2] R. Datko, "Two examples of ill-posedness with respect to small time delays in stabilized elastic systems," IEEE Transactions on Automatic Control, vol. 38, no. 1, pp. 163-166, 1993.

[3] A. Guesmia and S. A. Messaoudi, "General energy decay estimates of Timoshenko systems with frictional versus viscoelastic damping," Mathematical Methods in the Applied Sciences, vol. 32, no. 16, pp. 2102-2122, 2009.

[4] S. A. Messaoudi and B. Said-Houari, "Uniform decay in a Timoshenko-type system with past history," Journal of Mathematical Analysis and Applications, vol. 360, no. 2, pp. 459-475, 2009.

[5] Q. Y. Dai and Z. F. Yang, "Global existence and exponential decay of the solution for a viscoelastic wave equation with a delay," Zeitschrift Angewandte Mathematik Und Physik, vol. 65, no. 5, pp. 885-903, 2014.

[6] S. Nicaise and C. Pignotti, "Stabilization of second-order evolution equations with time delay," Mathematics of Control, Signals, and Systems, vol. 26, no. 4, pp. 563-588, 2014.

[7] S. Nicaise and C. Pignotti, "Exponential stability of abstract evolution equations with time delay," Journal of Evolution Equations, vol. 15, no. 1, pp. 107-129, 2015. 
[8] H. Wang and G. Q. Xu, "Exponential stabilization of 1-d wave equation with input delay," WSEAS Transactions on Mathematics, vol. 12, no. 10, pp. 1001-1013, 2013.

[9] G. Xu and H. Wang, "Stabilisation of Timoshenko beam system with delay in the boundary control," International Journal of Control, vol. 86, no. 6, pp. 1165-1178, 2013.

[10] X. F. Liu and G. Q. Xu, "Exponential stabilization for Timoshenko beam with distributed delay in the boundary control," Abstract and Applied Analysis, vol. 2013, Article ID 726794, 15 pages, 2013.

[11] S. Nicaise and C. Pignotti, "Stability and instability results of the wave equation with a delay term in the boundary or internal feedbacks," SIAM Journal on Control and Optimization, vol. 45, no. 5, pp. 1561-1585, 2006.

[12] S. Nicaise and C. Pignotti, "Stabilization of the wave equation with boundary or internal distributed delay," Differential and Integral Equations, vol. 21, no. 9-10, pp. 935-958, 2008.

[13] M. Krstic and A. Smyshlyaev, "Backstepping boundary control for first-order hyperbolic PDEs and application to systems with actuator and sensor delays," Systems \& Control Letters, vol. 57, no. 9, pp. 750-758, 2008.

[14] J. Y. Park, Y. H. Kang, and J. A. Kim, "Existence and exponential stability for a Euler-Bernoulli beam equation with memory and boundary output feedback control term," Acta Applicandae Mathematicae, vol. 104, no. 3, pp. 287-301, 2008.

[15] J. Liang, Y. Chen, and B.-Z. Guo, "A new boundary control method for beam equation with delayed boundary measurement using modified smith predictors," in Proceedings of the 42nd IEEE Conference on Decision and Control, vol. 1, pp. 809814, Maui, Hawaii, USA, 2003.

[16] Y. F. Shang, G. Q. Xu, and Y. L. Chen, "Stability analysis of EulerBernoulli beam with input delay in the boundary control," Asian Journal of Control, vol. 14, no. 1, pp. 186-196, 2012.

[17] Y. F. Shang and G. Q. Xu, "Stabilization of an Euler-Bernoulli beam with input delay in the boundary control," Systems \& Control Letters, vol. 61, no. 11, pp. 1069-1078, 2012.

[18] Z.-J. Han and G.-Q. Xu, "Output-based stabilization of EulerBernoulli beam with time-delay in boundary input," IMA Journal of Mathematical Control and Information, vol. 31, no. 4, pp. 533-550, 2013.

[19] K. Y. Yang, J. J. Li, and J. Zhang, "Stabilization of an EulerBernoulli beam equations with variable cofficients under delayed boundary output feedback," Electronic Journal of Differential Equations, vol. 75, pp. 1-14, 2015.

[20] B.-Z. Guo and K.-Y. Yang, "Dynamic stabilization of an EulerBernoulli beam equation with time delay in boundary observation," Automatica, vol. 45, no. 6, pp. 1468-1475, 2009.

[21] F.-F. Jin and B.-Z. Guo, "Lyapunov approach to output feedback stabilization for the Euler-Bernoulli beam equation with boundary input disturbance," Automatica, vol. 52, pp. 95-102, 2015.

[22] A. Pazy, Semigroups of Linear Operators and Applications to Partial Differential Equations, vol. 44 of Applied Mathematical Sciences, Springer, New York, NY, USA, 1983.

[23] T. Caraballo, J. Real, and L. Shaikhet, "Method of Lyapunov functionals construction in stability of delay evolution equations," Journal of Mathematical Analysis and Applications, vol. 334, no. 2, pp. 1130-1145, 2007. 


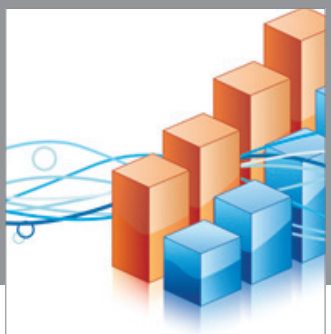

Advances in

Operations Research

vatem alat4

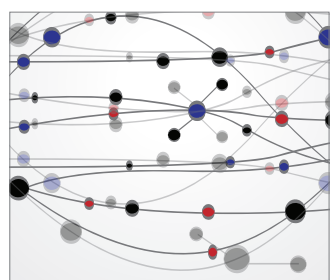

\section{The Scientific} World Journal
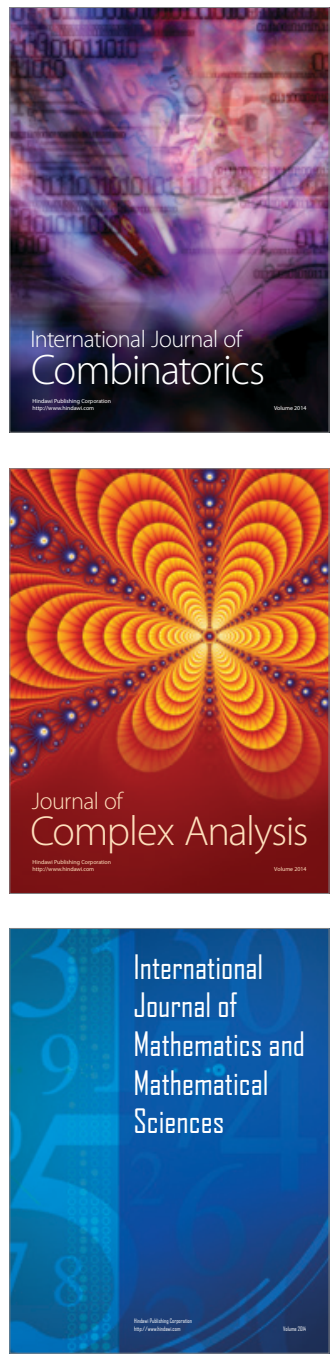
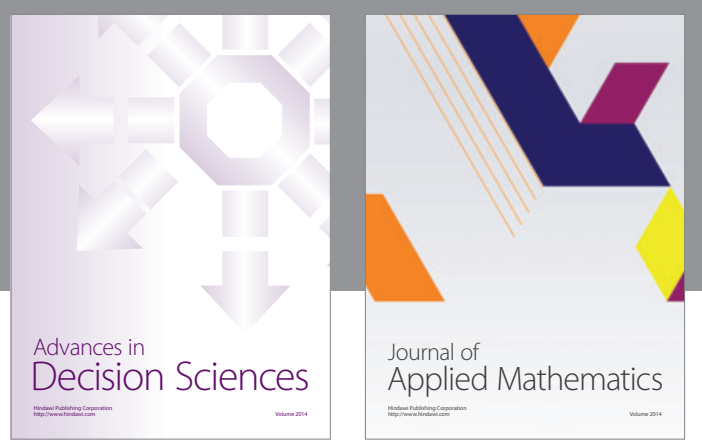

Algebra

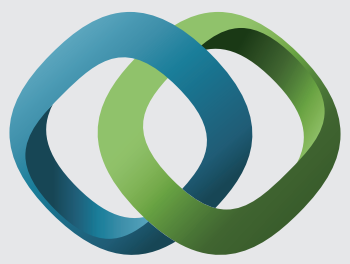

\section{Hindawi}

Submit your manuscripts at

http://www.hindawi.com
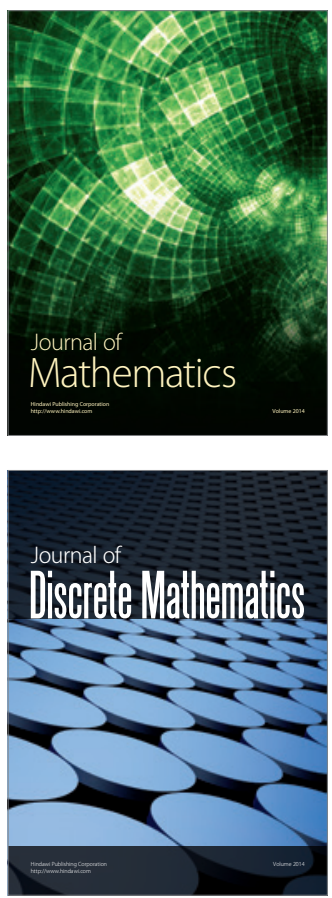

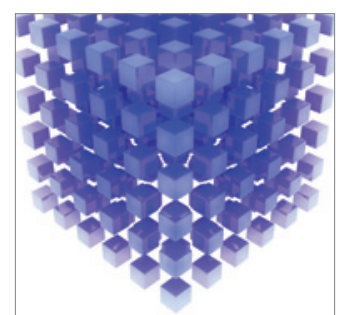

Mathematical Problems in Engineering
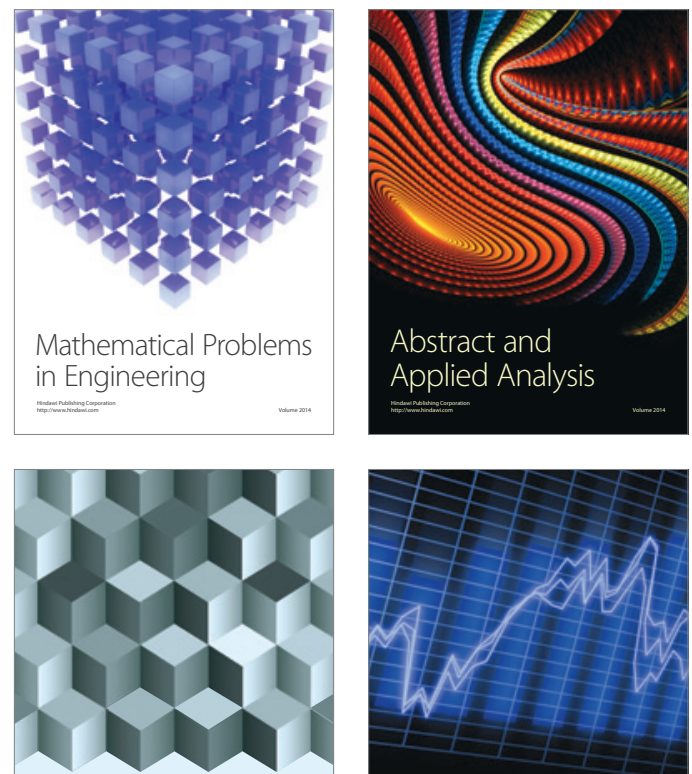

Journal of

Function Spaces

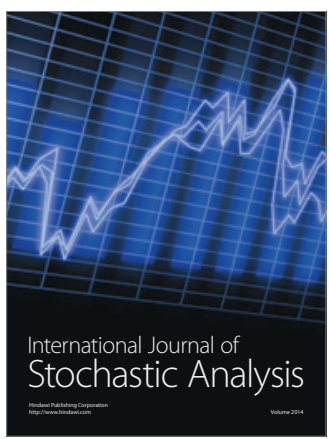

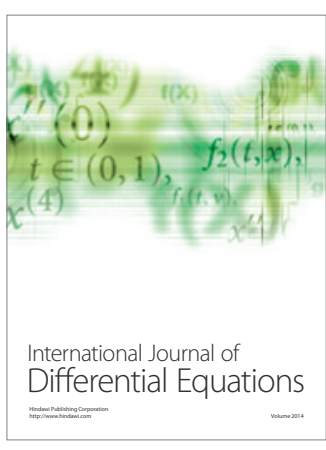
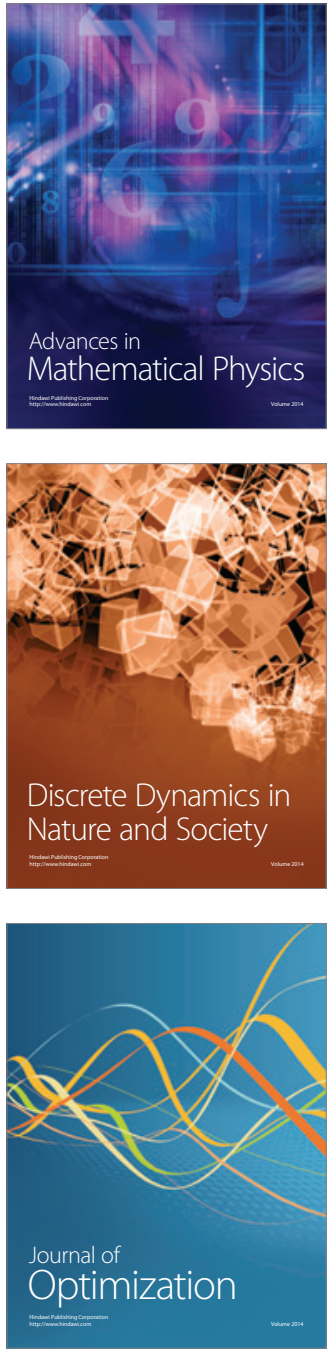